\title{
Competition Policy and Market Leaders
}

\author{
Ilir Maçi Krešimir Žigić \\ CERGE-EI, Prague CERGE-EI, Prague \\ Intertic
}

\begin{abstract}
We study a positive and normative aspect of a particular market setup in which there is initially a dominant firm that possesses technological and market leadership but eventually it ceases to be a leader after it is forced to reveal its patented superior technology to its competitors. Our analysis is motivated by the actual decision of the European Commission to impose a legal duty on a firm with a dominant position (Microsoft) to license its proprietary technology and intellectual property rights to its direct competitors. We show that under plausible assumptions like free entry or repeated market interactions there is a social value of market leadership and its mechanical removal by means of competition policy is likely to be harmful for society.
\end{abstract}

Keywords: Market Leaders, Competition Policy, Innovation

JEL: F12, F13, L11, L13, L16, K21 


\section{Introduction}

One of the key objectives of competition policy is to affect market structure and market conduct if they are deemed to be socially undesirable. When, for instance, market concentration exceeds a certain threshold, government usually undertakes measures to decrease the concentration by banning mergers or requiring large firms to divest. However, such an approach may under certain circumstances yield opposite outcome than desired. The reason is that the traditional approach in which usually the height of Herfindhal-Hirschman index determines whether market concentration is "excessive" or not is often too rough and it does not lie on solid theoretical grounds (see more on this in Motta, 2004).

Based on the rigorous game theoretic analysis, Sutton (1991) and Etro (2007) demonstrated that high market concentrations is in fact an outcome of tough (both price and non-price) competition rather than the indicator of market power and lack of competitive forces when conditions of free (or more generally, endogenous) entry prevail. (Note that the assumption of free entry is the reasonable one in characterizing of the long run equilibria). Thus, shifting market structure and related market conduct away from market leadership may have undesirable social effects in the dynamic markets (like, for example, software market) characterized by huge investment in R\&D and free entry. For instance, one way how the government can engineer such a shift in the software industry is to deprive the leading firm of its patented and widely spread product or from its superior technology by forcing it to reveal the secret pieces of information to its competitors. By revealing, say, source code of the most used operating system (through compulsory licensing) a "dominant" firm might be, among other things, stripped of its leading market position. So in the longer run there will be firms of similar power competing in the market. In the terms of market conduct, this situation could be described as a change from Stackleberg leadership to an "ordinary" oligopoly of firms with more even power.

In this paper we aim to study a positive and normative aspect of the above situation in which dominant firm is deprived of its leading position by means of competition policy. Our analysis is motivated by the actual decision of European Commission (EC) recently confirmed by the European Court of First Instances, to impose a legal duty on a firm with a dominant position (Microsoft) to license its proprietary technology and intellectual property rights (IPR) to its competitors so that they can incorporate that very same technology into their own directly competing products. This verdict is based on the reasoning that industry-wide innovation will be boosted in the longrun if the leading firm is deprived of its exclusive intellectual property rights. 
More specifically, according to EC this is justified when "on balance, the possible negative impact of an order to supply on dominant firm's incentives to innovate is outweighed by its positive impact on the level of innovation of the whole industry (including dominant firm)." Thus, the EC decision seems to establish a new balancing test under which the EC can order compulsory licensing. However, it seems that there was no underlying economic analysis on the side of EC that would support the above claims. Therefore, we reconsider this EC decision by relying on relevant economic analysis of market leaders in Section 2 (see Etro, 2006 and 2007) and also by relying on an equilibrium refinement in the repeated games that focuses on outcomes Preferred by Efficient Players (see Boone, 2002) in Section 3. Finally, in Section 4 we make a brief summary of our findings.

\section{Theory of Market Leaders: Cournot versus Stackelberg with R\&D and Free Entry}

In order to mimic the above situation where the leader is artificially deprived from its leading position, we first consider the ex post situation where firms are on an even technological level (symmetric Cournot equilibrium) and compare it with the ex ante (before enacted competition policy) situation when there exists a technological and market leader (Stackelberg equilibrium). We analyze both positive and normative aspect of these two different market environments, using a simple dynamic setup of two- and three-stage games where all firms invest in R\&D and where there is endogenous number of firms. The latter assumption captures the notion of long run equilibrium. We will only consider symmetrical equilibria.

Apart from a first mover advantage of the leader in the Stackelberg case, the markets are identical. The firms compete in quantities of imperfect substitutes. The inverse demand facing each firm $i$ is $P_{i}\left(q_{i}, q_{j}\right)=a-q_{i}-b \sum_{j \neq i} q_{j}$, where $b \in(0,1)$ captures the degree of substitutability. Furthermore, all firms must pay fixed setup cost $F>0$ to enter, and they incur $c-x_{i}$ marginal cost, where $c>0$ is constant and $x_{i}$ is R\&D investment of firm $i .^{1}$ The cost of this investment is $x_{i}^{2} / \gamma$, where $\gamma \in(0,1)$ measures the efficiency of R\&D.

\subsection{Cournot Competition}

The structure of the game in this environment is:

\footnotetext{
${ }^{1}$ Note that $x$ can also be interpreted as the investment in marketing and product development that enhances the size of the market captured by the parameter $a$.
} 
- There is a large number of potential entrants who decide whether to enter by incurring setup cost of $F$ or not.

- All entrants choose their investments $x_{i}$ and their output quantities $q_{i}$ simultaneously. So to simplify the analysis, we assume that R\&D investments are not chosen strategically to affect the subsequent competition in quantities but are determined in cost minimizing way. Allowing for this will make the analysis less transparent and will not change its main insights.

By backward induction we first find the optimal strategy of a firm if $n$ firms have decided to enter. After that we compute total output, price and profits to determine the equilibrium number of firms, $n^{*}$.

In the last stage each firm solves

$$
\max _{q_{i}, x_{i}} \Pi^{i}\left(q_{i}, x_{i}, q_{-i}\right)=\left(P_{i}-c+x_{i}\right) q_{i}-x_{i}^{2} / \gamma-F
$$

Taking the first order conditions of equation (1) and solving for symmetric output and investment we obtain

$$
q_{i}^{*}(n)=\frac{2(a-c)}{2 b(n-1)+4-\gamma}, \quad x_{i}^{*}(n)=\frac{\gamma(a-c)}{2 b(n-1)+4-\gamma}
$$

Notice that the levels of $q$ and $x$ are always proportional to each other in the equilibrium, namely $x_{i}=(\gamma / 2) q_{i}$. This result carries on to all firms in the market, hence it is also valid for aggregate market output and R\&D. Plugging (2) into the inverse demand and profit functions we can solve for $\pi_{i}^{C}$ as a function of $n$

$$
\pi_{i}^{C}(n)=\frac{(4-\gamma)(a-c)^{2}}{[2 b(n-1)+4-\gamma]^{2}}
$$

Finally, to find the equilibrium number of entrants we impose the condition that each firm's gross profit must justify its entry costs, that is, $\pi_{C}^{i}(n) \geq F$. For simplicity we will solve for a continuous $n^{*}$ and use equality

$$
n^{*}=\frac{(a-c) \sqrt{4-\gamma}-\sqrt{F}(4-\gamma-2 b)}{2 b \sqrt{F}}
$$

Hence, by plugging $n^{*}$ into (2) we can solve for equilibrium firm output and investment

$$
q_{i}^{*}=\frac{2 \sqrt{F}}{\sqrt{4-\gamma}}, \quad x_{i}^{*}=\frac{\gamma \sqrt{F}}{\sqrt{4-\gamma}}
$$


The corresponding market output and investment are

$$
\begin{aligned}
Q_{C}^{*} & =\frac{(a-c) \sqrt{4-\gamma}-\sqrt{F}(4-\gamma-2 b)}{b \sqrt{4-\gamma}} \\
X_{C}^{*} & =\frac{\gamma[(a-c) \sqrt{4-\gamma}-\sqrt{F}(4-\gamma-2 b)]}{2 b \sqrt{4-\gamma}}
\end{aligned}
$$

Finally, the equilibrium price charged by firm $i$ is given by

$$
P_{i}^{C}=c+\frac{\sqrt{F}(2-\gamma)}{\sqrt{4-\gamma}}
$$

In the next section we will solve for Stackelberg equilibrium and compare the outcomes with the ones we just found.

\subsection{Stackelberg Competition}

With Stackelberg competition one of the firms (the leader) has the privilege to move first and choose its investment and output. ${ }^{2}$ Formally, the timing of the game is now the following:

- Leader enters and pays setup cost, F, and immediately chooses investment $x_{l}$ and output $q_{l}$.

- The other firms, the followers, decide whether to enter by paying $F$ each.

- Those who enter decide on their $x_{i}$ and $q_{i}$ simultaneously.

By backward induction, we solve the followers' problem taking the leader's output $q_{l}$ and the number of followers, $m$, as given. After that we solve for $m$ as a function of the $q_{l}$ and finally we use this "response" of the number of entrants and each $q_{i}$ as conditions in the leader's problem. Hence, each follower's problem is

$$
\max _{q_{i}, x_{i}} \Pi^{i}\left(q_{i}, x_{i}, q_{-i}, q_{l}\right)=\left(P_{i}-c+x_{i}\right) q_{i}-x_{i}^{2} / \gamma-F
$$

Taking the first order conditions and solving for the symmetric equilibrium we get

$$
q_{i}^{*}\left(m, q_{l}\right)=\frac{2\left(a-c-b q_{l}\right)}{2 b(m-1)+4-\gamma}, \quad x_{i}^{*}\left(m, q_{l}\right)=\frac{\gamma\left(a-c-b q_{l}\right)}{2 b(m-1)+4-\gamma}
$$

\footnotetext{
${ }^{2}$ We assume that a monopoly always justifies its fixed cost so the first firm would always want to enter.
} 
We can now find the profit of each follower and solve for the number of followers as a function of the leader's strategy, $m\left(q_{l}\right)$. Much like in Section 1 , we use the zero profit condition to obtain

$$
m\left(q_{l}\right)=\frac{\left(a-c-b q_{l}\right) \sqrt{4-\gamma}-\sqrt{F}(4-\gamma-2 b)}{2 b \sqrt{F}}
$$

Not surprisingly, the number of followers falls with $q_{l}$, the more aggressively the leader behaves, the less place in the market for followers. It is interesting however, to see how the output of each firm changes with the leader's output, because there are two opposite effects at work. The first is the direct response effect because $\partial q_{i}^{*}\left(m, q_{l}\right) / \partial q_{l}$ is negative as seen from (7). However, at the same time an increase in leader's output reduces the numbers of followers in equilibrium and thus has positive effect on follower's output since $\left(\partial q_{i}^{*}\left(m, q_{l}\right) / \partial m\right)\left(d m / d q_{l}\right)>0$. We can plug (8) into (7) to get the net response in both follower strategies

$$
q_{i}^{*}\left(q_{l}\right)=\frac{2 \sqrt{F}}{\sqrt{4-\gamma}}, \quad x_{i}^{*}\left(q_{l}\right)=\frac{\gamma \sqrt{F}}{\sqrt{4-\gamma}}
$$

Two facts immediately hit the eye; first, the two above described effects exactly offsets each other so the followers' actions do not change with the leader's strategy. Moreover, their strategies (outputs) are the same as in the Cournot game under free entry that we solved earlier. The finding that equilibrium strategy of a follower is not affected by the leader's strategy when entry is free holds for a rather general setup and for a large variety of market conducts (see Etro, 2006). Hence, $q_{l}$ will only affect the total output of the followers through $m$, not $q_{i}^{*}$.

We can now come to the final set of equations that will be derived by the leader's problem

$$
\max _{q_{l}, x_{l}} \Pi^{l}\left(q_{l}, x_{l}\right)=\left\{\left[a-b m\left(q_{l}\right) q_{i}^{*}-q_{l}\right]-c+x_{l}\right\} q_{l}-x_{l}^{2} / \gamma-F .
$$

Taking first order conditions and solving them we get

$$
q_{l}^{*}=\frac{2(4-\gamma-2 b) \sqrt{F}}{(4-\gamma-4 b) \sqrt{4-\gamma}}, \quad x_{l}^{*}=\frac{\gamma(4-\gamma-2 b) \sqrt{F}}{(4-\gamma-4 b) \sqrt{4-\gamma}}
$$

Comparing the leader's output with the one of followers, we see that

$$
q_{l}^{*}=\frac{4-\gamma-2 b}{(4-\gamma-4 b)} q_{i}^{*}
$$


Given that $4-\gamma-4 b>0$, this implies that the leader behaves more aggressively than the followers. ${ }^{3}$

We can now also solve for the equilibrium number of followers $m^{*}$ by plugging $q_{l}^{*}$ in $m^{*}(8)$ and compute the difference between the Cournot number of firms $n^{*}$ and the Stackelberg $m^{*}+1$

$$
n^{*}-\left(m^{*}+1\right)=\frac{2 b}{4-\gamma-4 b}>0
$$

Hence, we have found that when one firm has a first mover advantage, we observe fewer firms in equilibrium. We must also compare the total output and investment with the Cournot case

$$
\begin{aligned}
Q_{S}^{*} & =\frac{(a-c) \sqrt{4-\gamma}-\sqrt{F}(4-\gamma-2 b)}{b \sqrt{4-\gamma}} \\
X_{S}^{*} & =\frac{\gamma[(a-c) \sqrt{4-\gamma}-\sqrt{F}(4-\gamma-2 b)]}{2 b \sqrt{4-\gamma}}
\end{aligned}
$$

Finally, to compare with (5), here are the equilibrium prices of follower $i$ and leader

$$
\begin{aligned}
P_{i}^{S} & =c+\frac{\sqrt{F}(2-\gamma)}{\sqrt{4-\gamma}} \\
P_{l}^{S} & =c+\frac{\sqrt{F}(4-\gamma-2 b)(2-\gamma-2 b)}{\sqrt{4-\gamma}(4-\gamma-4 b)}
\end{aligned}
$$

Much like Etro (2006), we also show that the aggregate output in Stackelberg setup is identical with the total output in the Cournot case when entry deterrence is not optimal. Furthermore, after comparing equations (5), (10) and (11), we see that the Stackelberg followers charge the same price as Cournot firms, while the leader charges a lower price. Hence, there are clear gains in consumer surplus. This result is formally shown in Appendix A.

Finally, note that the accommodation occurs in equilibrium for $b$ that is not "too large". In Appendix B we include some graphs that show this result. As is well known, when the products get less differentiated, the entry deterrence eventually becomes an optimal strategy. However, the threshold value of $b$ is, loosely speaking, larger compared to the standard Stackelberg framework with exogenously given number of followers. The reason is that, unlike in the standard Stackelberg setup, the leader's accommodation profit in the setup with endogenous entry is increasing in differentiation parameter

\footnotetext{
${ }^{3}$ This condition is also necessary for the leader's output and R\&D to be positive.
} 
$b .{ }^{4}$ The intuition is that, when products get more alike, competition becomes tougher, and, as a consequence, fewer firms enter in equilibrium. In other words, the leader can afford to squeeze out of the market more potential entrants as products become less differentiated. By the same token, the leader's accommodation profit increases in parameter $F$, since it also leads to a lower number of entrants in equilibrium.

\subsection{Long Run versus Short Run}

The above characterizations are aimed to portray two long-run equilibria: a Stackelberg as one before the policy implementation and a Cournot after the policy was in place for long time. However, we should also be able to tell more about the intermediate situation that occurs soon after the leader has been deprived of its position but before the industry adjusts to its long run equilibrium. This intermediate or short run situation can be described as Cournot equilibrium with exogenous number of firms. Recall that in a Stackelberg equilibrium there is one leader and $m^{*}$ followers. Now assume that as a result of the government intervention, the leader loses its advantage and, hence, the market transforms itself into a Cournot-like setup with $m^{*}+1<n^{*}$ firms. From the results in (2), treating the number of firms as exogenously set to $n=m+1$ one can clearly see that now each firm will produce less output and invest less intensively in R\&D compared to the setup with the leader and endogenous entry. However, there will be now positive profits but the total social welfare would be still lower than in the initial setup with the leader and free entry.

Thus, the considered action of the antitrust authorities will clearly help the competitors of (former) leader since they will now be able to generate positive profits (at least in the short run) while the consumers will be definitely worse off.

\section{$3 \quad$ Market Leadership in Repeated Interactions}

In the third section of the paper we consider a somewhat different market scenario. We now assume that there is repeated market competition in prices but the produced goods are assumed to be homogeneous. One of the firms has an advantage over the others in terms of R\&D productivity. We will call this firm the leader, because, although the game is played simultaneously each period, this advantage will enable it to assume leadership.

\footnotetext{
${ }^{4}$ See Dixit (1979) for the related comparative static results the in the standard Stackelberg setup.
} 
To be more precise, all firms who enter choose a price $p_{i}$ and a level of R\&D expenditure $x_{i}$. Each firm faces a constant marginal cost $c_{i}\left(x_{i}\right)$, where $c_{i}>0, c_{i}^{\prime}<0$ and $c_{i}^{\prime \prime}>0$ at all levels of $x_{i}$. The leader's cost function differs from the other firms by $c_{L}(x)<c_{F}(x)$ for all $x>0$ and $c_{L}(0)=c_{F}(0)$. The demand is $D(p)$ where $D^{\prime}<0$. Finally, the firms compete in prices over an infinity of periods and discount the future by a rate $r$. In such setup, the Folk theorem predicts that there is a multitude of potential equilibria. However, Boone (2002) argues that casual observation and theoretical and empirical evidence suggests that when there are significant differences in efficiency levels among players, the most efficient players act aggressively and impose an outcome that is more beneficial for them. Therefore he proposes an equilibrium refinement that focuses on outcomes Preferred by Efficient Players (PEP). Strategies associated with the PEP refinement distinguish between two types of deviations: 1 . slight undercuts of the price by a firm to gain the whole market at the expense of its opponents, and 2. reductions in price which, if followed, increase the profits of all firms with positive profits. In the former case firms retaliate to punish the deviator; in the latter case deviator's price cut is followed by all remaining firms. Thus, in the second case, deviators act as market leaders.

When the collusive outcome is modeled using the PEP refinement, the toughness of market competition is driven endogenously by the level of heterogeneity that exists among firms (in our case, by the distribution of firms' unit costs). If firms have similar levels of cost efficiency, there is a "balance of power" inducing firms to behave "softly" towards each other and charge high prices. In this situation any price undercut is perceived as the first type of deviation and leads to the Bertrand Nash outcome. On the contrary, if some firms are much more efficient than their competitors, it pays to price aggressively and drive the less efficient firms out of the market. The price undercut is then perceived as the second type of deviation and is not met by retaliation from the surviving firms.

\subsection{Market without Leader}

Much like in the previous section, we are interested to see what happens in this market if the leader loses its advantage over other firms. If all firms are identical there is a perfect "balance of power", so the firms will reach a tacit collusion and the above PEP refinement suggests that they will charge a monopoly price. ${ }^{5}$ We now assume that the cost function is the same $c_{L}\left(x_{i}\right)$

\footnotetext{
${ }^{5}$ Such a collusive outcome can also be sustained in standard infinitely repeated games by grim trigger strategy if the discount $r$ is low enough.
} 
for all firms. ${ }^{6}$ Under this strategy, all firms keep to the monopoly price unless one of them undercuts it. In that case all charge $p=c(x)$ for all remaining periods, that is, the equilibrium reverts to standard Bertrand outcome on the market with homogenous goods. Hence, each potential deviant is caught between a full monopoly profit in this period and none afterwards or an infinite stream of shared monopoly profits. ${ }^{7}$ For the collusion equilibrium to be sustainable and assuming that there are $n$ identical firms we need

$$
D\left(p^{m}\right)\left[p^{m}-c_{L}\left(x_{i}\right)\right] / n-x_{i} \geq r D\left(p^{m}\right)\left[p^{m}-c_{L}\left(x_{i}\right)\right]-x_{i}
$$

which holds if and only if $r \leq 1 / n$. We will assume henceforth that this condition holds, that is, a market where all firms are identical would result in them charging monopoly price $p^{m}$. Each firm chooses a level of $\mathrm{R} \& \mathrm{D} x^{a}$ that maximizes

$$
x^{a}=\arg \max _{x}\left\{D\left(p^{m}\right)\left[p^{m}-c_{L}(x)\right] / n-x\right\}
$$

Taking the first order conditions we find the following rule that implicitly defines $x^{a}$

$$
c_{L}^{\prime}\left(x^{a}\right)=-\frac{n}{D\left(p^{m}\right)}
$$

\subsection{Market with Leader}

Now we return to the assumption that one firm, the leader, has a cost advantage for all positive values of $x$. Assuming $x_{L}$ is positive in equilibrium, if this advantage is large enough, it would be optimal for the leader to charge a price that would exclude all $n$ followers and still make a profit. The leader would have to charge a price $p^{d}$ that is defined by

$$
\max _{x}\left\{D\left(p^{d}\right)\left[p^{d}-c_{F}(x)\right]-x\right\}=0
$$

But even if charging $p^{d}$ and selling to the whole market produces a positive profit, it would have to produce more profit than the alternative solution: accommodating the followers' entry and sharing the monopoly profits. Hence, the leader will deter entry if and only if

$$
\max _{x}\left\{D\left(p^{d}\right)\left[p^{d}-c_{L}(x)\right]-x\right\} \geq \max _{x}\left\{D\left(p^{m}\right)\left[p^{m}-c_{L}(x)\right] / n-x\right\}
$$

\footnotetext{
${ }^{6}$ The same outcome will be supported by PEP even if firms have different unit costs with variance lower than the critical level that triggers aggressive behavior of the more efficient firm(s).

${ }^{7}$ The deviant would only undercut marginally therefore getting (slightly less than) a monopolist's profits for a single period.
} 
Again, for the sake of our argument, we will assume this condition to hold. That is, if there is a firm that has a (large enough) cost advantage, it will deter all the other firms from entry charging $p^{d}<p^{m}$ and assume the market leadership position.

The leader's optimal choice of R\&D in these circumstances is

$$
x^{d}=\arg \max _{x}\left\{D\left(p^{d}\right)\left[p^{d}-c_{L}(x)\right]-x\right\}
$$

The first order condition gives us the implicit rule for the optimal R\&D

$$
c_{L}^{\prime}\left(x^{d}\right)=-\frac{1}{D\left(p^{d}\right)}
$$

Comparing equations (13) and (16) we can see that $x^{d}>x^{a}$ because $c_{L}^{\prime}\left(x^{d}\right)$ is equated to a smaller (in absolute value) number than $c_{L}^{\prime}\left(x^{a}\right)$ and $c_{L}^{\prime \prime}>0 .^{8}$ However, it remains unclear whether the single firm spends more in $\mathrm{R} \& \mathrm{D}$ or less than the $n$ collusive firms. That is, whether $x^{d}>n x^{a}$ or the other way around depends on the properties of $c_{L}(x)$ and the demand function. In the case of linear demand and the standard "R\&D production function" displaying decreasing returns (like the one of the form $c_{L}(x)=$ $c-\sqrt{\gamma x}$ ), the setup with the technological leader and $n-1$ followers results in higher R\&D investment and larger innovation than the corresponding symmetric setup without the leader. ${ }^{9}$ Furthermore, as proven in Appendix $\mathrm{D}$, a sufficient condition for the leader spending more in $\mathrm{R} \& \mathrm{D}$ than the firms is that the elasticity of $c_{L}^{\prime}(x) \in[-1,0)$.

\section{Conclusion}

The main message of our analysis is that under plausible assumptions like free entry or repeated market interactions there is a social value of market leadership and its mechanical removal by means of competition policy is likely to be harmful for society. As stated in Economic Focus of The Economist sometime ago "... antitrust authorities should be especially careful when trying to stamp out monopoly power in markets that are marked by technical innovation. It could still be that firms like Microsoft are capable of using their girth to squish their rivals; the point is that continued monopoly is not cast-iron evidence of bad behavior [...] The fact that a dominant firm remains on top might actually be strong evidence of vigorous competition.

\footnotetext{
${ }^{8}$ The number is smaller for two reasons. First, the numerator of the derivative of (13) is $n$ instead of 1 . Second, its denominator $D\left(p^{m}\right)$ is lower than $D\left(p^{m}\right)$ because $p^{m}>p^{d}$.

${ }^{9}$ See Appendix C.
} 
[...] The very ease of entry, and the aggressiveness of the competitive environment, are what spur monopolists to innovate so fiercely." ("Slackers or Pace-setters," 2004)

In Section 2 we showed that Stackelberg leadership outcome mimics that of Cournot but with a smaller number of firms. This corresponds to a higher social welfare in Stackelberg leader setup due to fewer setup costs to be paid. Moreover, in our setup, R\&D investments are always proportional to outputs in equilibrium. This ensures that the Stackelberg equilibrium also replicates Cournot outcomes in aggregate investment levels. Furthermore, we have also shown that there is social welfare loss in the aftermath of applied policy that removes the leadership position. The implied structure and conduct change induced by the antitrust policy is not neutral in terms of market price and quantities. As the industry moves from one long run equilibrium to the other, output and investment are lower and the price is higher. Consequently, the only beneficiaries of such a policy are the competitors that benefit at the expense of consumers and the leader.

In Section 3 we study the positive and normative aspects of competition in prices when there are repeated interactions among the potentially different firms. We show that when there is a distinctive technological leader, it converts its technological advantage into market leadership. The leader behaves aggressively, charges lower price and (under plausible conditions) invests more in $R \& D$ than would be the case in a similar setup without the technological and market leader. As a consequence, entry is deterred and the followers are forced to leave the market. 


\section{References}

Boone, J. (2002). Balance of power. CEPR Discussion Paper 4733.

Dixit, A. (1979). A model of duopoly suggesting a theory of entry barriers. Bell Journal of Economics, 10(1), 20-32.

Etro, F. (2006). Aggressive leaders. The Rand Journal of Economics, 37(1), $146-154$.

Etro, F. (2007). Competition, innovation and antitrust: A theory of market leaders and its policy implications. Berlin \& New York: Springer Verlag.

Motta, M. (2004). Competition policy: Theory and practice. Cambridge: Cambridge University Press.

Slackers or pace-setters? Monopolies may have more incentives to innovate than economists have thought. (2004, May 22). The Economist, Economic Focus.

Sutton, J. (1991). Sunk cost and market structure. Massachusetts: MIT Press. 


\section{A The Difference in Consumer Surplus with and without Leader}

In this appendix we show that the consumer surplus is greater in the free entry Stackelberg equilibrium than in the Cournot equilibrium. Since we are dealing with several horizontally differentiated markets we have to add the surplus of each market. We will compute consumer surplus by the area under each demand function.

Due to the symmetry between firms, in Cournot equilibrium (section 2.1) we have

$$
C S^{C}=n^{*} \int_{P_{i}^{C}}^{P_{0}} D_{i}\left[P_{i}, Q_{-i}\right] d P_{i}
$$

where $D_{i}$ is the demand facing firm $i$ that depends on its own price $P_{i}$ and the equilibrium output of all other firms $Q_{-i}$. The price where $D_{i}=0$ is denoted by $P_{0}$.

By the same token, the total consumer surplus in Stackelberg equilibrium (section 2.2) is given by

$$
C S^{S}=m^{*} \int_{P_{i}^{S}}^{P_{0}} D_{i}\left[P_{i}, Q_{-i}\right] d P_{i}+\int_{P_{l}^{S}}^{P_{0}^{l}} D_{l}\left[P_{l}, m^{*} q_{i}^{*}\right] d P_{l}
$$

where $D_{l}$ is the leader's demand and $P_{0}^{l}$ is the price where $D_{l}=0$. The price $P_{0}$ and $Q_{-i}$ are the same as for firm $i$ in the Cournot setup because in equilibrium their individual outputs as well as the total industry output are identical (compare $q_{i}^{*}$ from sections 2.1 and 2.2 and also $Q_{C}^{*}$ with $Q_{S}^{*}$ ). Indeed, the consumer surplus generated by each firm (product) is the same for firm $i$ in Cournot equilibrium and follower $i$ in Stackelberg equilibrium.

Hence, the difference between the total consumer surplus under each setup (expression (18) less expression (17)) is

$$
C S^{S}-C S^{C}=\left(m^{*}-n^{*}\right) \int_{P_{i}^{S}}^{P_{0}} D_{i}\left[P_{i}, Q_{-i}\right] d P_{i}+\int_{P_{l}^{S}}^{P_{0}^{l}} D_{l}\left[P_{l}, m^{*} q_{i}^{*}\right] d P_{l} .
$$

After some straightforward algebra this expression is reduced to

$$
C S^{S}-C S^{C}=\frac{4 b F(4-\gamma-2 b)}{(4-\gamma)(4-\gamma-4 b)^{2}}
$$

which is always positive for our initial condition $4-\gamma-4 b>0$. 


\section{B Complete vs. Partial Entry Deterrence}

In an ednogenous entry setting, like our section 2 model, the leader always manages the number of followers to some extent. Therefore, in these cases we always have deterrence of some competitors. What we intend to discuss in this appendix is whether the leader would prefer to deter entry completely, that is, not allow any followers to enter. For simplicity we will only refer to this scenario as entry deterrence.

We will compare the profit of the maximizing leader (internal solution) to the profit of the leader who maximizes his profit by producing enough output to make it unprofitable for even one follower to enter (corner solution). Formally, to find the entry deterring output from the leader we use $q_{l}^{D}$ that solves

$$
m\left(q_{l}^{D}\right)=1
$$

because, by definition of $m(\cdot)$, that will set the profit of a single follower to zero.

The algebraic expressions, while well defined and straightforward to compute, are too cumbersome to be represented here, therefore we will limit ourselves with including some graphs of the difference between profit levels as a function of the differentiation parameter $b$. Figure 1 below shows the difference in profit (internal less corner) as a function of $b$ for the following parameters $a=10, F=3, c=2$ and for $g=1$ (left) and $g=1.5$ (right)
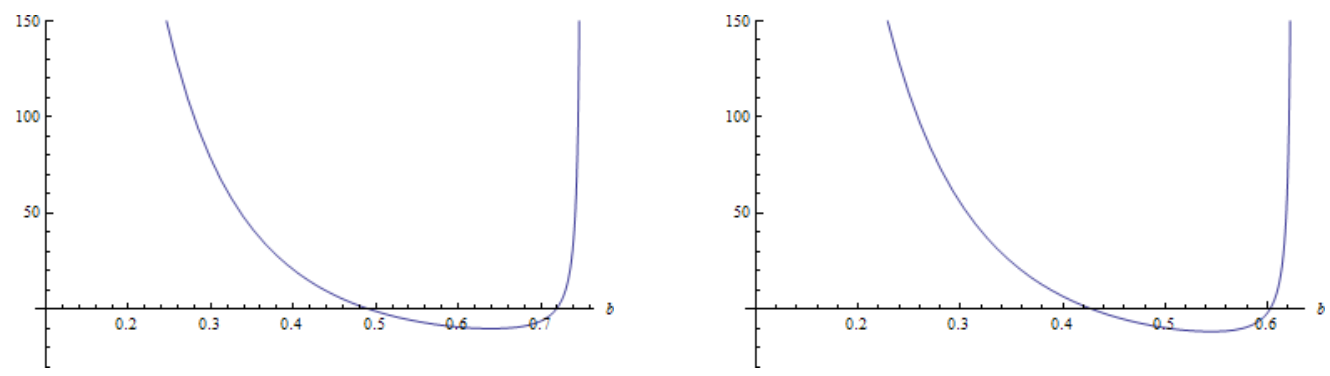

Figure 1: Difference in profit of leader

As it is clearly seen for the graphs, the leader chooses to allow for some entrants when $b$ is smaller than some limit value. Our analysis in section 2 is valid for these values of $b$ where there is no complete entry deterrence. 


\section{Repeated Price Competition with Linear Demand and Quadratic Research Costs}

In this appendix we show that in the special case of linear demand and quadratic research costs (that we adopted in section 2) the single deterring firm in repeated price competition setting ("leader" of section 3) will spend more in R\&D than the symmetric equilibrium.

In order to make the models comparable we need to compute the R\&D production function that leads to the quadratic costs. We have to do this because in section $2 x_{i}$ represents the amount of research (the fall in production costs) whereas in section $3 x_{i}$ refers to the R\&D expenditure.

From section 2 the cost of decreasing the production marginal cost by $x$ is $x^{2} / \gamma$. Inverting this to get a production function (and redefining $x$ as $R \& D$ expenditure to fit section 3 ) we find that by spending $x$ a firm will have marginal production cost of $c-\sqrt{\gamma x}$.

Using this R\&D technology and a linear demand, the symmetric firm in a market with $n$ firms will maximize

$$
\max _{x_{i}} \Pi^{i}=\left(a-p^{m}\right)\left[p^{m}-\left(c-\sqrt{\gamma x_{i}}\right)\right] / n-x_{i}-F .
$$

We set the monopoly price to $p^{m}=\left[a-\left(c-\sqrt{\gamma x_{i}}\right)\right] / 2$ and solve the first order condition to get the optimal expenditure

$$
x^{a}=\frac{\gamma(a-c)^{2}}{(4 n-\gamma)^{2}} .
$$

In the case when one firm has an advantage in R\&D technology, it may decide to keep every other firm out of the market. In our example we will assume these other firms (from here "followers") have $c-\sqrt{\gamma_{f} x_{i}}$ production costs for $x_{i}$ spent on research, where $\gamma_{f}<\gamma^{10}$ When this deterrence is optimal the leader will solve

$$
\max _{x_{L}} \Pi^{L}=\left(a-p^{d}\right)\left[p^{d}-\left(c-\sqrt{\gamma x_{L}}\right)\right]-x_{L}-F
$$

where $p^{d}<p^{m}$ is the deterrence price, that is, the market price that makes $\Pi^{i}=0$. Taking the first order condition and solving it we get

$$
x^{d}=\frac{\gamma\left\{2(a-c)\left[a-c+\sqrt{(a-c)^{2}-F\left(4 n-\gamma_{f}\right)}\right]-F\left(4 n-\gamma_{f}\right)\right\} n^{2}}{\left(4 n-\gamma_{f}\right)^{2}}
$$

which is greater than $n x^{a}$.

\footnotetext{
${ }^{10} \mathrm{We}$ assume the other firms should have a disadvantage rather than the leader having an advantage in order to get a result that differs from the symmetric case only due to market structure rather than superior technology.
} 


\section{A Sufficient Condition for Higher R\&D Expenditure by Single Firm}

For R\&D to be higher under a single (leader) firm than under $n$ identical collusive firms we need $x^{d}>n x^{a}$. Futhermore, as already discussed in the paper, at optimum

$$
n\left|c_{L}^{\prime}\left(x^{d}\right)\right| \leq\left|c_{L}^{\prime}\left(x^{a}\right)\right|
$$

To simplify our analysis, we ignore the difference in denominators between (13) and (16). The previous inequality in that case holds with equality. This condition is weaker than what we already have because a lower $\left|c_{L}^{\prime}\left(x^{d}\right)\right|$ would imply an even bigger $x^{d}$. Thus, for the leader to be producing more we would need that at least an $n$ times higher $x$ is necessary to produce an $n$ times lower $\left|c_{L}^{\prime}(x)\right|$. Formally

$$
\frac{\left|c_{L}^{\prime}\left(x_{1}\right)\right|}{\left|c_{L}^{\prime}\left(x_{2}\right)\right|} \leq \frac{x_{2}}{x_{1}}
$$

must hold for all $0<x_{1}<x_{2}$. Taking the log of both sides and rearranging

$$
-\left[\log \left|c_{L}^{\prime}\left(x_{2}\right)\right|-\log \left|c_{L}^{\prime}\left(x_{1}\right)\right|\right] \leq \log x_{2}-\log x_{1}
$$

For infinitesimal differences between $x_{2}$ and $x_{1}$ we have

$$
\epsilon_{c_{L}^{\prime}}=\frac{d \log \left|c_{L}^{\prime}(x)\right|}{d \log x} \geq-1
$$

One type of marginal cost function that yields this result is $c_{L}(x)=$ $a-b x^{1+\epsilon}$ where $\epsilon \in(-1,0)$ is the desired (constant) elasticity of $c_{L}^{\prime}(x)$. For a unit elasticity $c_{L}^{\prime}(x)$ the function is $c_{L}(x)=a-b \ln x$. These marginal cost functions are not positive everywhere but $a$ and $b$ can be set such that the marginal cost is positive for all the relevant levels of $R \& D$. 